\title{
Captura incidental en nasas del pez león Pterois volitans (Scorpaeniformes: Scorpaenidae) en el Caribe colombiano
}

\author{
Merlys Merlano Cera ${ }^{1} \&$ Rocío García-Urueña ${ }^{1}$ \\ 1. Grupo de Investigación de Ecología y Diversidad de Algas Marinas y Arrecifes Coralinos, Universidad del Magdalena, \\ Carrera 32 No 22-08 Santa Marta, Colombia; merlysmmc@gmail.com, rgarciau@unimagdalena.edu.co
}

Recibido 29-III-2018. Corregido 28-V-2018. Aceptado 29-VI-2018.

\begin{abstract}
Incidental catch in traps of the lionfish Pterois volitans (Scorpaeniformes: Scorpaenidae) in the Colombian Caribbean. Incidental catch in traps of the lionfish Pterois volitans (Scorpaeniformes: Scorpaenidae) in the Colombian Caribbean. Since 2011, small-scale fishermen from Taganga Bay (Colombian Caribbean) have been catching lionfish in traps in the Tayrona National Natural Park, which had caused them to believe that the lionfish is the cause of the decrease in overall fishing. In order to determine the changes in the species captured and to know the abundance of the lionfish in this fishing method, we analyzed the historical landings in traps between 1993 and 2014, as well as the landing of three fishing units between March 2012 and July 2014. The historical analysis showed that the decline in species and kilograms landed has been occurring since 2000. A total of 4913 specimens were landed between March 2012 and July 2014, of which 85 \% were species of commercial interest, and the rest were used by fishermen for consumption. Landings between 2012 and 2014 were conditioned by climatic conditions, where the co-occurrence between Pterois volitans and the main commercial species and self-consumption did not provide evidence of the lionfish affecting its abundance. This confirms the presence of the lionfish as a new member in the landings in traps the Taganga Bay. Rev. Biol. Trop. 66(3): 1197-1207. Epub 2018 September 01.
\end{abstract}

Key words: lionfish; commercial fish; landings; fishermen; biological invasions.

La invasión biológica en el Caribe del pez león Pterois volitans (Linnaeus, 1758) ha sido exitosa (Albins \& Hixon, 2008), con fuertes efectos sobre las comunidades arrecifales (Sutherland et al., 2010; Albins \& Hixon, 2011). En Colombia fue registrado en el 2008 para la isla de San Andrés, Providencia y Santa Catalina (Abril-Howard et al., 2013) y desde el 2009 para el Caribe continental (González, Grijalba-Bendeck, Acero, \& Betancur, 2009; Arbeláez \& Acero, 2011; Hernández-Abello, García-Urueña, \& Acero, 2015; Coronado-Carrascal, García-Urueña, \& Acero, 2015; García-Urueña, Acero, \& Coronado-Carrascal, 2015). Se cree que a futuro los impactos del invasor tendrían un efecto negativo en la pesquería, el turismo y la industria del acuario; al reducirse los desembarcos de especies de importancia económica (Morris \& Whitfield, 2009).

A ésta problemática se suma la sobrepesca, el aumento en la presión pesquera, la introducción de artes nocivas, la contaminación y la pesca industrial de arrastre, como factores que en el Caribe han causado el declive en las capturas (Salas, Chuenpagdee, Seijo, \& Charles, 2007; Manjarrés et al., 2008; Grijalba-Bendeck, Novoa, Bustos-Montes, PosadaPeláez, \& Santafé Muñoz, 2012). Por ejemplo, García (2008) menciona que los pescadores artesanales del Caribe colombiano, años atrás extraían cerca de 20 especies de importancia comercial pertenecientes a familias como Lutjanidae, Scombridae, Carangidae, Centropomidae, Serranidae, Haemulidae y que ahora son escasas; además las horas de pesca y la 
profundidad de captura han aumentado. Para el margen costero del Magdalena en la década de 1990, el registro de la captura anual era de aproximadamente 2240 t (Manjarrés, 2004), mientras que para el 2012 se registró un total de 1671.6 t (Bustos-Montes et al., 2012). Para la bahía de Taganga los registros de desembarcos anuales en nasas superaban los $240 \mathrm{~kg}$ entre 1994 y 2008, que luego en el 2010 disminuyeron a aproximadamente $61 \mathrm{~kg}$ (Manjarrés, 2004; Grijalba-Bendeck et al., 2012).

Para el caso de los pescadores artesanales de la bahía de Taganga registran desde el 2011 la presencia de $P$. volitans en nasas que son distribuidas en áreas del Parque Nacional Natural Tayrona (PNNT), por lo cual, han atribuido la disminución de sus capturas a la presencia del pez león. En esta bahía, la pesca artesanal es una de las actividades más importante para sus pobladores, debido a su práctica ancestral y a los aportes económicos (Bustos-Montes et al., 2012), siendo el arte más utilizado el chinchorro, aunque la línea de mano, las redes de enmalle, los palangres y las nasas también son frecuentes (Grijalba-Bendeck et al., 2012). Éstas últimas son construidas por lo general para la captura de crustáceos y peces de importancia comercial (Nédélec \& Prado, 1990), siendo Lutjanus synagris, L. analis, Calamus penna y Haemulon plumierii las más importantes (Barros \& Manjarrés, 2004b).

Debido a que los pescadores en este importante puerto pesquero atribuyen la disminución en sus capturas en nasas a la presencia del pez león, se propuso recopilar la información bibliográfica del desembarco histórico en este arte de pesca para la Bahía de Taganga, analizar el desembarque de distintas unidades económicas de pesca (UEP) en nasas mediante encuestas después de la invasión y examinar si la presencia del pez león es realmente el detonante en la reducción de las capturas.

\section{MATERIALES Y MÉTODOS}

La bahía de Taganga se encuentra ubicada al Norte de Santa Marta, entre Punta Venados (11 ${ }^{\circ} 16^{\prime} 14^{\prime \prime} N$ \& $\left.74^{\circ} 12^{\prime} 16^{\prime \prime} \mathrm{W}\right)$, Punta Colorada
(11 15 '44" N \& 74¹1'56" W) (Rubio-Polanía \& Trujillo-Arcila, 2013). En esta bahía desembarcan las unidades económicas de pesca (UEP) que utilizan nasas y realizan sus faenas en el PNNT.

Análisis del desembarque histórico en nasas: Se analizó la información del desembarque en nasas para la zona de estudio consignada en Grijalba-Bendeck et al. (2012), la cual detalla en términos de kilogramos por faena la captura entre octubre de 2008 a junio de 2010; mientras la información de Manjarrés (2004) y Anónimo (2014), detalla valores de kilogramos y las especies desembarcadas en nasas en los periodos de 1993 a 2000 y de 2012 a 2014, respectivamente, conteniendo datos estandarizados del esfuerzo de muestreo. Con los datos obtenidos se realizaron análisis descriptivos.

DPUE (individuos/faena) entre marzo 2012 y julio 2014: Entre marzo y noviembre de 2012 se encuestó una UEP y luego entre agosto 2013 y julio 2014 se encuestaron tres UEP, a las que se le registró características como el número de individuos desembarcados (discriminada por especie) y el número de nasas revisadas en cada faena. Se realizó la estandarización a una unidad (total de individuos de cada especie/ total nasas revisadas en la faena), debido a que las UEP realizan sus faenas con diferentes cantidades de nasas $(7,12,21,39$ etc.). Se determinó la talla y peso de los peces león en cada desembarco. Con estas variables se determinó el DPUE que incluye el esfuerzo pesquero involucrado y los individuos/faena (De la Hoz, Manjarres-Martínez, \& Cuello, 2015).

El total del desembarco fue separado en dos componentes, aquello que es comercializado y lo que los pescadores utilizan para el autoconsumo de su núcleo familiar y/o lo que regalan. Para analizar el DPUE de los peces de importancia comercial por mes se realizó un análisis de varianza de una vía (número de individuos/faena) luego de comprobar los supuestos de homogeneidad de varianza y normalidad. Por la concordancia de la toma de datos en la bahía de Taganga con los registros 
de peces comerciales, obtenidos por el Sistema de Información Estadístico Pesquero Colombiano (Anónimo, 2014), estos fueron incluidos como una muestra en el análisis.

\section{Desembarco del pez león (individuos/} faena) entre marzo 2012 y julio 2014: la coocurrencia del pez león con respecto a las especies de interés comercial y las del autoconsumo, fue evaluada mediante estadística descriptiva. Adicionalmente, a través de regresión lineal se examinó tanto la relación entre la abundancia del pez león con el conjunto de todas las especies desembarcadas, como de los desembarcos coincidentes entre las especies comerciales y de autoconsumo con el pez león.

Para analizar la variación del tamaño de los peces león desembarcados durante los diferentes periodos de muestreo, se realizó una ANOVA a dos vías entre años de desembarco (2012-2014) y las cuatro categorías de talla en que fueron separados los peces (categoría 1 de 100-199 mm, categoría 2 de 200-299 mm, categoría 3 de 300 a 399 mm y categoría 4 de más de $400 \mathrm{~mm}$ ). El análisis se realizó usando el programa Statgraphics.

\section{RESULTADOS}

Desembarque histórico: el desembarque total entre 1994 y 1996 superó los $240 \mathrm{~kg}$, a diferencia de 1993 y 2000 cuando fue menor a 52 y $20 \mathrm{~kg}$ respectivamente. Las principales especies capturadas fueron Lutjanus synagris, Calamus penna y Panulirus argus. Para el 2008 se obtuvo un total de $41.1 \mathrm{~kg}, 126.6 \mathrm{~kg}$ en el 2009 y $61 \mathrm{~kg}$ en el 2010 (Cuadro 1), con Lutjanus vivanus y L. purpureus como las especies más representativas. En el 2013 se obtuvo un total de $107.7 \mathrm{~kg}$, mientras en 2012 y 2014 el aporte fue inferior a los $50 \mathrm{~kg}$ (Cuadro 1), registrando a L. synagris como la especie de mayor desembarque (Apéndice).

Se resalta a 1996 con la mayor captura $(233.43 \mathrm{~kg}$ ) donde la familia Lutjanidae fue la más representativa, mientras que el 2000 mostró la menor captura $(9 \mathrm{~kg})$ y a partir del 2008 se observa valores que no superaron los

\section{CUADRO 1}

Desembarcos mensuales ( $\mathrm{kg}$ ) de la pesca artesanal con nasas en la bahía de Taganga por periodos, entre mayo 1993 y abril 2014

TABLE 1

Monthly landings $(\mathrm{kg})$ of artisanal fishing with pots in Taganga Bay in different periods, between May 1993 and April 2014

\begin{tabular}{lccccccccccc}
\multicolumn{1}{c}{ Mes/Año } & \multicolumn{4}{c}{ Manjarres (2004) } & \multicolumn{4}{c}{ Grijalba-Bendeck et al. (2012) } & \multicolumn{2}{c}{ Anónimo (2014) } \\
& 1993 & 1994 & 1995 & 1996 & 2000 & 2008 & 2009 & 2010 & 2012 & 2013 & 2014 \\
Enero & - & - & 33.6 & 50.4 & - & - & - & 23.00 & - & 5 & - \\
Febrero & - & 7.5 & 16.4 & 27.63 & - & - & - & 2.50 & - & 5.75 & 27.5 \\
Marzo & - & 11.2 & 14.3 & 59.7 & - & - & 5.50 & 14.00 & - & - & - \\
Abril & - & 24.2 & 25.4 & 28.75 & - & - & 6.90 & 6.30 & - & 8.5 & 18.5 \\
Mayo & 12.2 & - & 21.2 & 18.85 & - & - & 20.20 & 12.00 & - & - & - \\
Junio & - & 47.9 & 16.8 & 56.75 & 7.9 & - & 19.80 & 3.00 & - & - & - \\
Julio & - & 16.9 & 24.2 & 29.52 & - & - & 10.40 & - & - & 30 & - \\
Agosto & 12.3 & 64.2 & 27.7 & 29.5 & - & - & 4.80 & - & - & 20 & - \\
Septiembre & 5.7 & 22.2 & 18.1 & 24.47 & - & - & 8.10 & - & - & - & - \\
Octubre & 9 & 19.1 & 6.8 & 19.8 & 4.5 & 13.50 & 22.50 & - & - & - & - \\
Noviembre & 3.5 & 10.5 & 43.1 & 30.6 & 2.5 & 27.60 & 12.50 & - & 29 & 26,5 & - \\
Diciembre & 8.8 & 16.9 & - & 22.58 & - & - & 15.40 & - & 13 & 12 & - \\
Total & 51.5 & 240.6 & 247.6 & 398.55 & 14.9 & 41.1 & 126.1 & 60.8 & 42.0 & 107.75 & 46.0 \\
\hline
\end{tabular}


$65.5 \mathrm{~kg}$. Es importante mencionar que especies como Carangoides bartholomaei, Lutjanus griseus y Haemulon plumierii, eran poco capturadas entre 1993 y 1996 (Manjarrés, 2004), que luego para el 2000 ya no fueron registradas. $\mathrm{Su}$ ausencia también se ratifica para los datos de Grijalba-Bendeck et al. (2012) y Anónimo (2014) (Apéndice).

DPUE (individuos/faena) entre 2012 y 2014: Se observó que en el segundo semestre de 2012 y 2013 se presentaron mayores valores de DPUE en comparación con el primer semestre, a diferencia de marzo de 2014. Para el 2012 y el 2013 los mayores registros de DPUE fueron en noviembre seguido de septiembre y octubre; mientras que en 2014 fueron en marzo, abril y febrero (Cuadro 2). En total se registró un desembarque de 4913 individuos, de los cuales el $85 \%$ fueron especies comerciales (4 179), principalmente L. analis (944), L. synagris (936), C. penna (512) y la langosta P. $\operatorname{argus}(891)$. El $15 \%$ fue autoconsumo (728)

\section{CUADRO 2}

Desembarco total de la pesca artesanal con nasas (\#individuos/faena), obtenido de las encuestas a las unidades económicas de pesca entre marzo 2012 y julio 2014

TABLE 2

Total landings of artisanal fishing with traps (\#ind./chore), obtained from surveys at economical Fishing units between March 2012 and July 2014

\begin{tabular}{lccc}
\multicolumn{1}{c}{ Mes/Año } & 2012 & 2013 & 2014 \\
Enero & - & 0.7 & 4.5 \\
Febrero & - & 3.7 & 10.6 \\
Marzo & 0.5 & - & 34.5 \\
Abril & 7.5 & 1.5 & 15.1 \\
Mayo & 8.2 & - & 6.7 \\
Junio & 5.5 & - & 9.7 \\
Julio & 8.0 & 3.6 & 6.2 \\
Agosto & 5.3 & 3.6 & - \\
Septiembre & 10.5 & 7.0 & - \\
Octubre & 9.2 & 8.5 & - \\
Noviembre & 19.8 & 9.3 & - \\
Diciembre & 2.2 & 6.1 & - \\
Total & 76.7 & 44 & 87.3 \\
Porcentaje & $36.8 \%$ & $21.1 \%$ & $41.9 \%$ \\
\hline
\end{tabular}

y lo constituyo P. volitans (265), Haemulon aurolineatum (154), Sparisoma viride (79) y Carpilius corallinus (64).

Los peces comerciales correspondieron a 16 especies, de las cuales 7 pertenecen a las familias Lutjanidae y el resto Labridae, Carangidae, Sparidae Priacanthidae y Haemulidae. Se encontró diferencias significativas en el DPUE para los peces de interés comercial (ANOVA, $\mathrm{P}=0.0000$ ), siendo $L$. analis, $L$. synagris y $C$. penna aquellas con mayores desembarques. Carangoides bartholomaei, Sparisoma aurofrenatum, Lutjanus apodus y Seriola rivoliana fueron las menos representativas. En cuanto a su variación mensual no hubo diferencias significativas, sin embargo, en 2012 las principales capturas fueron en mayo y noviembre. Para 2013 se presentaron picos en agosto y noviembre y para 2014 en marzo, abril y junio.

El DPUE mensual de la coocurrencia del pez león, mostró que fue desembarcado en todo el 2012, siendo más representativo en septiembre y noviembre, mientras que el componente comercial y el autoconsumo fue en mayo y abril respectivamente. En 2013 el pez león superó lo comercial en cerca del $40 \%$ en septiembre y al autoconsumo de agosto-diciembre. Para el 2014 nuevamente el pez león fue superior a lo comercial en marzo y abril ( $5 \%$ ), aunque estuvo ausente en febrero y mayo. Mientras que el autoconsumo fue más representativo en junio y julio en comparación con el desembarque del pez león. Específicamente para el pez león el DPUE promedio se duplicó año a año (2012: 0.11 - 2013: 0.24 - 2014: 0.48) (Fig. 1).

El análisis de regresión del pez león con el conjunto de todas las especies desembarcadas no mostró ningún patrón claro. Luego, al examinar la abundancia entre el pez león y las principales especies de los dos componentes, se encontró la tendencia donde $P$. volitans superó a $H$. aurolineatum y a $H$. cruentatus, siendo significativa sólo para $H$. aurolineatum $(\mathrm{F}=$ 3.23; $\mathrm{P}=0.0705)$. Sin embargo, el $\mathrm{R}^{2}$ ajustado solo explicó hasta un $19 \%$ de la variabilidad y no son significativos; por lo cual se considera que no hay ningún patrón que demuestre que 


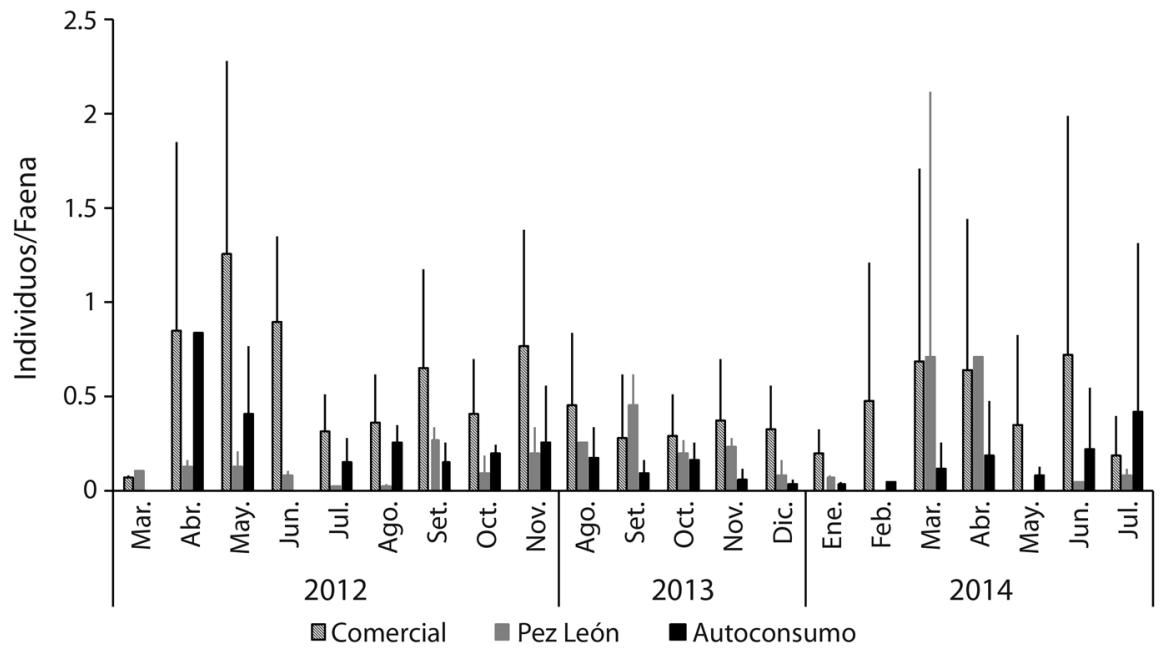

Fig. 1. Relación de la co-ocurrencia del pez león con el componente comercial y el autoconsumo entre marzo 2012 y julio 2014 (líneas indica desviación estándar).

Fig. 1. Relationship of the co-occurrence of lionfish with commercial component and the self-consumption between March 2012 and July 2014.

P. volitans afecte la presencia de las especies analizadas (Fig. 2).

La talla de los peces león $(n=265)$ osciló entre 130 y $420 \mathrm{~mm}$ de longitud total con un promedio de $264 \mathrm{~mm}$, los peces de la categoría $4(>400 \mathrm{~mm})$ fueron pocos $(\mathrm{n}=12)$, mientras que los de la categoría $2(200-299 \mathrm{~mm})$ fueron los de mayor desembarque $(\mathrm{n}=127)$. En 2012 y 2013 hubo mayor número de individuos con tallas entre 200-299 mm en comparación con el 2014 donde los ejemplares en su mayoría presentaron una talla entre $300-399 \mathrm{~mm}$. Sin embargo, no se encontró diferencias significativas entre las categorías de talla $(\mathrm{P}=0.35) \mathrm{ni}$ para los años $(\mathrm{P}=0.72)$.

\section{DISCUSIÓN}

La pesca artesanal al igual que la industrial ha sido afectada principalmente por los nocivos artes que se han implementado, con capturas inferiores a la talla de madurez (García, Pérez, Duarte, \& Manjarrés, 2008; Manjarrés et al., 2008), pues solo en el Caribe colombiano la captura total marina pasó de 11022 t en 2006 a 2966 en el 2009 (Grijalba-Bendeck et al.,
2012). Específicamente para la pesca artesanal con nasas en la bahía de Taganga, se observó en el análisis histórico, que desde el 2000 la lista de especies inició su descenso y luego para el 2010 se evidenció para el total en kilogramos desembarcados (Manjarrés, 2004; GrijalbaBendeck et al., 2012; Anónimo, 2014). Páez (2011) menciona para la parte norte del Caribe colombiano al uso de la dinamita, la creciente demanda en el mercado y la contaminación, como las posibles causas de la reducción de los kilogramos desembarcado.

En cuanto a las especies que constituyen el desembarco, el pescador típico del Caribe colombiano percibe como escasas a L. synagris, Ocyurus chrysurus, Scomberomorus regalis, $C$. bartholomei y P. argus, así como la escases de los volúmenes de su captura (García, 2008). A la vez, especies que anteriormente eran descartadas como el sable y el pez loro, son ahora de interés, llegando a remplazar aquellas consideradas de importancia comercial (García, 2008; Barros \& Manjarrés 2004b). Grijalba-Bendeck et al. (2012) y Anónimo (2014) soportan estas observaciones, ya que especies como L. griseus, y L. mahogoni desde el 2000 no presentan registro de su desembarco. 

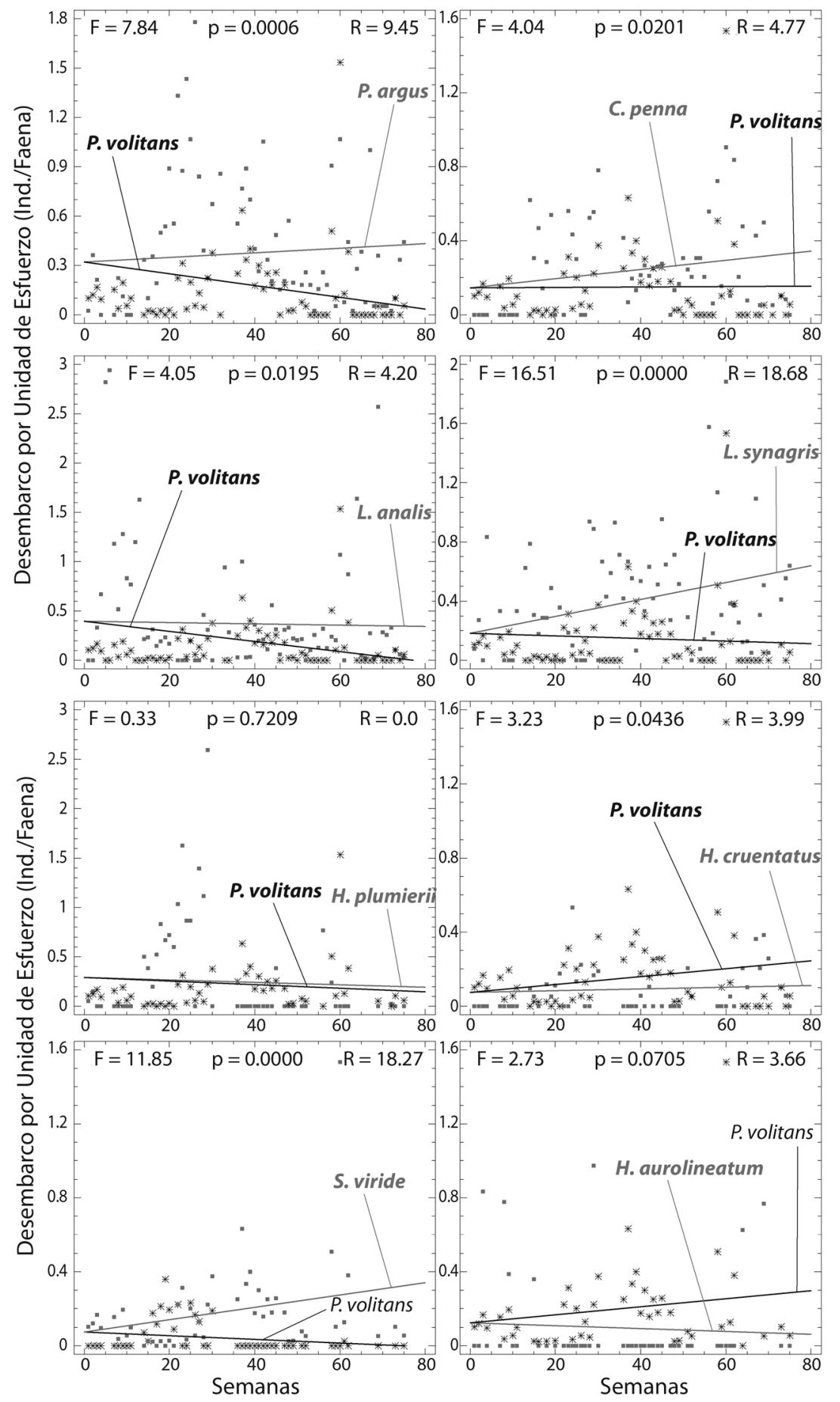

Fig. 2. Relación de la co-ocurrencia entre Pterois volitans y las principales especies capturadas en nasas entre marzo 2012 y agosto 2014 ( $\mathrm{n}=75$ semanas; (0-30: 2012), (31-50: 2013) y (51-75: 2014).

Fig. 2. Relationship of the co-occurrence between Pterois volitans and the main species captured in traps between March 2012 to August 2014 ( $\mathrm{n}=75$ weeks; (0-30: 2012), (31-50: 2013) and (51-75: 2014). 
Manjarrés, Infante, Rueda y Escorcia (1993) mencionan que los desembarcos a inicio de año están asociados al fenómeno de surgencia, la cual se produce en La Guajira y alcanza la costa noreste de Santa Marta, lo que favorece la alta disponibilidad del recurso pesquero. Sin embargo, esto no coincidió para la DPUE en nasas entre 2012 y 2014 durante los primeros meses del año (enero-febrero), debido que las UEP redujeron su número de faenas de pesca, ya que las condiciones de vientos fuertes impidieron las operaciones de pesca (ob. per). Barros y Manjarrés (2004b) encontraron los valores mínimos de desembarco en enero y febrero 2000 y Bustos-Montes et al. (2012), igualmente encontraron el menor desembarco en enero 2010. Lozano-Duque et al. (2010) mencionan que entre marzo y agosto se presenta el periodo de transición de la zona de convergencia intertropical; lo cual favorece que las UEP puedan realizar más faenas en comparación con los primeros meses del año.

Para los componentes de la pesca dentro de las especies de interés comercial $(85 \%)$ se destacó $L$. analis, L. synagris. P. argus y C. penna correspondiendo al $66.72 \%$ del total desembarcado. Torres-Rodríguez (2010) encontró en la composición del desembarco de nasas para la bahía de Taganga a L. synagris con un $79.3 \%$, seguido de L. analis con el $18.1 \%$, para un total del $97.3 \%$. Manjarrés et al. (1993) exponen que en zonas del PNNT se da una disponibilidad relativamente alta de pargos durante todo el año, e igualmente para el Magdalena se ha encontrado que los mayores volúmenes de desembarcos en nasas corresponden a la familia Lutjanidae (51.2 \%) (Barros \& Manjarrés, 2004b). Bustos-Montes et al. (2012) encontraron a L. vivanus, L. purpureus, L. analis y L. synagris como especies predominantes, mientras Barros y Manjarrés (2004a) a L. synagris, L. analis, C. penna y algunos hemúlidos. Aunque los hemúlidos son mencionados por Barros y Manjarrés (2004a), solo se encontró a H. plumierii y su desembarco presentó un valor intermedio en el DPUE.

Se resalta la sustitución de especies ya que entre 2012 y 2014 se encontró a $S$. aurofrenatum, L. vivanus y C. bartholomaei como especies de interés comercial. Manjarrés (2004) presenta solo un registro de S. aurofrenatum y se consideraba una especie poco importante; mientras que Grijalba-Bendeck et al. (2012) y Anónimo (2014) no describen registro alguno de esta especie. Heteropriacanthus cruentatus que solo presenta registro en 1994 (Manjarrés, 2004) desde 2012 se hace evidente su desembarco. Por el contrario $C$. bartholomaei y L. vivanus que anteriormente se capturaban en grandes volúmenes, hoy día son escasos. Otras especies como Trichiurus lepturus y $S$. viride en la última década pasaron a ser especies de interés comercial (García, 2008; Saza, 2011).

Dentro del autoconsumo (15\%), para García (2008) este componente corresponde al 1.8 \% y Páez (2011) menciona que los productos de la pesca artesanal en su mayoría son para el consumo interno y la subsistencia. Entre marzo 2012 y julio 2014 se encontró a $P$. volitans, $H$. aurolineatum, C. crysos, C. corallinus, $S$. viride y Octopus vulgaris, como aquellas especies que sustentaron la alimentación de los pescadores y lo que regalan. Se resalta la inclusión del $P$. volitans en la pesca con nasas ya que representó el $5.41 \%$ del total desembarcado, mientras que para $H$. aurolineatum y C. corallinus fue de $3.14 \%$ y $1.30 \%$ respectivamente.

El pez león presentó una variación mensual irregular, siendo coincidente con el DPUE de los componentes comercial y el autoconsumo en abril y noviembre. Estudios de estadios de madurez, agregaciones, épocas de desove, etc., son numerosos para muchas especies ícticas, pero es escasa información sobre la relación que pueda tener el pez león con respecto a dichas variables. Por ejemplo, Bayraktarov, Alarcón-Moscoso, Polanco y Wild (2014) resaltan la escasez de Epinephelus striatus como un posible controlador biológico en el PNNT, lo cual podría favorecer la abundancia del pez león. Coronado-Carrascal et al. (2015), analizan la abundancias de ocho especies de importancia comercial y ecológica en relación a la presencia del pez león en áreas protegidas del PNNT y áreas expuestas; donde no se 
encontraron diferencias en zonas protegidas, pero si bajas abundancias en zonas expuestas para las especies de importancia comercial. Igualmente, García-Urueña et al. (2015) analizaron la comunidad íctica en cinco localidades del PNNT y arrecifes aledaños, donde encontraron a $P$. volitans entre las 20 especies más abundantes, con ninguna especie de importancia comerciales que lo superara en talla. Por lo cual, se requiere de estudios del cómo la presencia del pez león puede influir en la ecología arrecifal.

Lesser y Slattery (2011) y Morris y Whitfield (2009) concuerdan en que los peces león tienen la capacidad de afectar la estructura y funcionamiento de poblaciones marinas, llegando a transformar la trama alimentaria al reducirse los organismos herbívoros que mantienen controlada las macroalgas. Además, se cree que, si las poblaciones de las presas preferidas (Labridae, Pomacentridae, Gobiidae y Serranidae) del pez león se agotan con el tiempo, es posible que se concentre en los juveniles de especies de interés comercial (Morris \& Akins 2009; Morris \& Whitfield, 2009). El modelo de regresión de $P$. volitans fue analizado solo en relación a la captura principalmente de especies comerciales y éste, no explicó ningún patrón del que se pudiera inferir afectación a dichas especies. Se resalta el hecho que las nasas son revisadas cada 5 o 7 días, donde se reduce la disponibilidad de alimento y por ende la valoración que se puede hacer sobre afectaciones en la trama alimenticia.

Las tallas de los peces león oscilaron entre 130 y $420 \mathrm{~mm}$ de LT con un promedio de $264 \mathrm{~mm}$, siendo mayores a las registradas mediante capturas con arpón para el Caribe colombiano, las cuales varían entre 64 y 265 $\mathrm{mm}$ de longitud total y de 183 a $363 \mathrm{~mm}$ longitud estándar (González, Acero, Serrat-LL, \& Betancur, 2011; Bayraktarov et al., 2014; Hernández-Abello et al., 2015). Bayraktarov et al. (2014) evaluaron la longitud del pez león en cuatro bahías del PNNT y encontraron la mayor talla $(400 \mathrm{~mm})$ en Cinto y Neguanje, también registraron que el $75 \%$ de los peces observados presentaban tallas entre 200 y 250 $\mathrm{mm}$ de LT, lo cual es coincidente con las tallas en 2013 (200-299 mm).

Los registros del DPUE del pez león en nasas permiten confirmar su presencia en este arte de pesca, establecido como un nuevo integrante del componente para autoconsumo, superando al pulpo y al cangrejo pero no a las especies de interés comercial y la langosta. Por lo cual, los registros de este estudio no consideran la presencia del pez león en el arte de pesca con nasas, como el detonante en la reducción de las especies que constituyen el desembarco para la bahía de Taganga, puesto que desde el 2000, cuando no existía la invasión, se evidencian cambios en la composición de especies y los volúmenes desembarcados.

\section{AGRADECIMIENTOS}

A la comunidad de pescadores en la Bahía de Taganga y a Luis Manjarrés por la asesoría estadística.

\section{RESUMEN}

Desde el 2011, los pescadores artesanales de Taganga (Caribe colombiano) capturaron al pez león en nasas para el Parque Nacional Natural Tayrona. Este hecho generó la percepción de que el pez león causó la disminución de la pesca. Para determinar los cambios en las especies capturadas y conocer la abundancia del pez león en este arte, se analizó el desembarco histórico en nasas entre 1993 y 2014, así como, el desembarque a tres unidades económicas de pesca entre marzo 2012 y julio 2014. El análisis histórico mostró que la disminución de las especies y los kilogramos desembarcados ocurrió desde el 2000. Un total de 4913 individuos fueron desembarcados entre marzo 2012 y julio 2014, de los cuales el $85 \%$ fueron especies de interés comercial y el resto fueron utilizadas por los pescadores para su consumo. Los desembarcos entre marzo 2012 y julio 2014 estuvieron condicionados a factores climáticos, donde la co-ocurrencia de Pterois volitans y las principales especies comerciales y de autoconsumo no mostró evidencia de que el pez león afecte sus abundancias. Se confirma así la presencia del pez león como un nuevo integrante en los desembarcos en nasas en la Bahía de Taganga.

Palabras clave: pez león; peces comerciales; desembarcos; pescador; invasiones biológicas 


\section{REFERENCIAS}

Abril-Howard, A., Bolaños-Cubillos, N., Llanos-Ruiz, C., Taylor, E., González, A., Polanco, A., ... Diavanera, A. (2013). Protocolo para la captura, extracción y disposición final del pez león (Pterois volitans) en Colombia. Convenio No. 023-2012 suscrito entre Ministerio de Ambiente y Desarrollo Sostenible MADS y la Corporación para el Desarrollo Sostenible del Archipiélago de San Andrés, Providencia y Santa Catalina: CORALINA.

Albins, M. A., \& Hixon, M. A. (2008). Invasive IndoPacific lionfish Pterois volitans reduce recruitment of Atlantic coral-reef fishes. Marine Ecology Progress Series, 367, 233-238.

Albins, M. A., \& Hixon, M. A. (2011). Worst case scenario: potential long-term effects of invasive predatory lionfish (Pterois volitans) on Atlantic and Caribbean coral reef communities. Environmental Biology of Fishes, 641, 95-101.

Anónimo. (2014). Boletín estadístico SEPEC período noviembre de 2012 - diciembre de 2014. Colombia: Autoridad Nacional de Acuicultura y Pesca (AUNAP).

Arbeláez, N., \& Acero, A. (2011). Presencia del pez león Pterois volitans (Linnaeus) en el manglar de la Bahía de Chengue, Caribe Colombiano. Boletín de Investigaciones Marinas y Costeras, 40(2), 431-435.

Barros, M., \& Manjarrés, L. (2004a). Inventario y caracterización general de la flota pesquera artesanal del sector norte del departamento del Magdalena (Taganga - La Jorará). En L. Manjarrés (Ed.), Pesquerías demersales del área norte del Mar Caribe de Colombia y parámetros biológico-pesqueros y poblacionales del recurso pargo (pp. 13-22). Santa Marta: Universidad del Magdalena.

Barros, M., \& Manjarrés, L. (2004b). Recursos pesqueros explotados por las pesquerías artesanales marítimas del sector de Taganga - La Jorará (dpto. del Magdalena), con énfasis en peces demersales. En L. Manjarrés (Ed.), Pesquerías demersales del área norte del Mar Caribe de Colombia y parámetros biológico pesqueros y poblacionales del recurso pargo (pp. 55-76). Santa Marta: Universidad del Magdalena.

Bayraktarov, E., Alarcón-Moscoso, J., Polanco, A., \& Wild, C. (2014). Spatial and temporal distribution of the invasive lionfish Pterois volitans in coral reefs of Tayrona National Natural Park, Colombian Caribbean. Peer Journal, 2, 397.

Bustos-Montes, D., Cuello, F. J., Salas-Castro, S., Saza, A., De la Hoz, J., Grijalba-Bendeck, M., ... Cuellar-Pinzón, J. (2012). Variación espacio-temporal del desembarco pesquero artesanal marítimo del departamento del Magdalena (junio 2008 - junio 2010). En M. Grijalba-Bendeck, D. Bustos-Montes, C. Posada-Peláez, \& A. Santafé-Muñoz (Eds.), La pesca artesanal maritima del departamento del Magdalena una visión desde cuatro componentes (pp. 43-114). Colombia: Universidad de Bogotá Jorge Tadeo Lozano.

Coronado-Carrascal, V., García-Urueña, R., \& Acero, A. (2015). Comunidad de peces arrecifales en relación con la invasión del pez león: el caso del Caribe sur. Boletín de Investigaciones Marinas y Costeras, 44(2), 291-302.

De la Hoz-M., J., Manjarres-Martínez, L., \& Cuello, F. (2015). Estadísticas de desembarco, esfuerzo, valor monetario y rentas de la pesquería artesanal de Taganga periodo abril-diciembre de 2015. Bogotá: Autoridad Nacional de Acuicultura y Pesca.

García, C. B. (2008). Conocimiento tradicional: lo que los pescadores artesanales del Caribe colombiano tienen para decirnos. Pan-Americana Journal of Aquatic Science, 5(1), 78-90.

García, C. B., Pérez, D., Duarte, L. O., \& Manjarrés, L. (2008). Experimental results with a reducing device for juvenile fishes in a tropical shrimp fishery: impact on the invertebrate bycatch. Pan-Americana Journal of Aquatic Science, 3(3), 275-281.

García-Urueña, R., Acero, A., \& Coronado-Carrascal, V. (2015). Comunidad de peces arrecifales en presencia del pez león (Pterois volitans) en Santa Marta, Caribe colombiano. Revista MVZ Córdoba, 20, 4989-5003.

González, J., Grijalba-Bendeck, M., Acero, A., \& Betancur-R., R. (2009). The invasive red lionfish, Pterois volitans (Linnaeus 1758), in the southwestern Caribbean Sea. Aquatic Invasions, 4(3), 507-510.

González-C., J., Acero, A., Serrat-LL., A., \& Betancur-R., R. (2011). Caracterización taxonómica de la población del pez león Pterois volitans (Linnaeus 1758) (Scorpaenidae) residente en el Caribe colombiano: merística y morfometría. Biota Colombiana, 2, 15-22.

Grijalba-Bendeck, M., Novoa, A., Bustos-Montes, D., Posada-Peláez, C., \& Santafé Muñoz, A. (2012). Elementos de manejo y conservación de los recursos ícticos aprovechados para la pesca marítima artesanal que se efectúa en el margen costero del departamento del Magdalena, Caribe Colombiano. En M. GrijalbaBendeck, D. Bustos-Montes, C. Posada-Peláez \& A. Santafé-Muñoz (Eds.), La pesca artesanal marítima del departamento del Magdalena una visión desde cuatro componentes (pp. 417-454). Colombia: Universidad de Bogotá Jorge Tadeo Lozano.

Hernández-Abello, J., García-Urueña, R., \& Acero, A. (2015). Estructura de tallas y preferencia al sustrato del pez león (Pterois volitans) (Scorpaeniformes: Scorpaenidae) en Santa Marta (Colombia). Acta Biológica Colombiana, 20(2), 175-182.

Lesser, M., \& Slattery, M. (2011). Phase shift to algal dominated communities at mesophotic depths associated 
with lionfish (Pterois volitans) invasion on a Bahamian coral reef. Biological Invasions, 13, 1855-1868.

Lozano-Duque, Y., Medellín-Mora, J., \& Navas, G. (2010). Contexto climatológico y oceanográfico del mar caribe colombiano. En INVEMAR (Eds.), Biodiversidad del margen continental del Caribe colombiano (pp. 52-84). Colombia: Serie de Publicaciones Especiales, Invemar, 20.

Manjarrés, L (Ed). (2004). Estadísticas pesqueras artesanales del Magdalena y la Guajira, con aplicación de herramientas informáticas para su sistematización y procesamiento. Santa Marta: Unimag-Incoder-Inpa-Colciencias.

Manjarrés, L., Duarte, L. O., Altamar, J., Escobar, F., García, C., \& Cuello, F. (2008). Effects of using bycatch reduction devices on the Colombian Caribbean Sea shrimp fishery. Ciencias Marinas, 34(2), 223-238.

Manjarrés, L., Infante, J., Rueda, A., \& Escorcia, F. (1993). Carta pesquera del área de Santa Marta. En Informe técnico final. Proyecto integral de investigaciones y desarrollo de la pesca artesanal marítima en el área de Santa Marta (pp. 45-54). Santa Marta, Colombia: INPA-CIID- UNIMAGDALENA.

Morris, J. A., \& Akins, J. L. (2009). Feeding ecology of invasive lionfish (Pterois volitans) in the Bahamian archipelago. Environmental Biology of Fishes, 86, 389-398.

Morris, J. A., Jr., \& Whitfield, P. E. (2009). Biology, Ecology, Control and Management of the Invasive IndoPacific Lionfish: An Updated Integrated Assessment. Washington, DC: NOAA Technical Memorandum NOS NCCOS 99.

Nédélec, C., \& Prado, J. (1990). Definición y clasificación de las diversas categorías de arte de pesca. Roma: FAO.
Páez, Y. (2011). Caracterización de las pesquerías artesanales entre tasajera y la bahía de santa marta (magdalena - caribe colombiano) considerando aspectos bioeconómicos, marzo - octubre 2009. Universidad de Bogotá Jorge Tadeo lozano, Santa Marta, Colombia. Recuperado de http://unicornio.utadeo.edu.co/ tesis/biologia_marina/T979.pdf

Rubio-Polanía, J. C., \& Trujillo-Arcila, C. A. (2013). Granulometric characteristics of the soft bottoms in an area of the Taganga bay (Colombia) influenced by the instalation of bentonic shelters. Revista U.D.C.A Actualidad y Divulgación Científica, 16(1), 193-203.

Salas, S., Chuenpagdee, R., Seijo, J., \& Charles, A. (2007). Challenges in the assessment and management of small-scale fisheries in Latin America and the Caribbean. Fisheries Research, 87, 5-16.

Saza, M. (2011). Análisis bioeconómicos de la actividad pesquera artesanal íctica en el departamento del Magdalena durante el 2009. Universidad de Bogotá Jorge Tadeo lozano, Santa Marta, Colombia. Recuperado de http://unicornio.utadeo.edu.co/tesis/biologia_marina/T979.pdf

Sutherland, W., Clout, M., Côté, I. M., Daszak, P., Depledge, M. H., Fellman, L., ... Watkinson, A. R. (2010). A horizon scan of global conservation issues for 2010 . Trends in Ecology and Evolution, 25(1), 1-7.

Torres-Rodríguez, J. (2010). Aspectos bioeconómicos de la pesca marina artesanal en el norte del departamento del magdalena entre marzo y octubre de 2009, Caribe Colombiano. Universidad de Bogotá Jorge Tadeo lozano, Santa Marta, Colombia. Recuperado de http:// unicornio.utadeo.edu.co/tesis/biologia_marina/T934. pdf 
APÉNDICE

Desembarcos mensuales $(\mathrm{kg})$ de las principales especies capturadas en nasas en la bahía de Taganga en diferentes periodos, entre mayo de 1993 y abril de 2014

\section{APÉNDICE}

Monthly landings $(\mathrm{kg})$ of the main species caught in pots in the bay of Taganga in different periods, between May of 1993 and April of 2014

\begin{tabular}{|c|c|c|c|c|c|c|c|c|c|c|c|}
\hline \multirow{2}{*}{ Familia } & \multirow{2}{*}{ Especie/Año } & \multicolumn{5}{|c|}{ Manjarres (2004) } & \multirow{2}{*}{$\begin{array}{l}\text { Grijalba- } \\
\text { Bendeck } \\
\text { et al. }(2012) \\
2008-2010\end{array}$} & \multicolumn{3}{|c|}{ Anónimo (2014) } & \multirow{2}{*}{ Promedio } \\
\hline & & 1993 & 1994 & 1995 & 1996 & 2000 & & 2012 & 2013 & 2014 & \\
\hline \multirow[t]{6}{*}{ Lutjanidae } & L. synagris & 21.6 & 84.1 & 112.7 & 79.0 & 7.9 & 9.2 & 21.5 & 33.3 & 14.8 & 42.7 \\
\hline & L. griseus & 2.0 & & 17.1 & & & & & & & 9.6 \\
\hline & L. analis & 0.6 & 3.8 & & 502 & 0.2 & 18.4 & 5.0 & 22.3 & 25.0 & 15.7 \\
\hline & L. mahogoni & & & & 2.7 & 0.1 & & & & 2.0 & 1.6 \\
\hline & L. vivanus & & & & & & 33.2 & 13.0 & & & 23.1 \\
\hline & L. purpureus & & & & & & 22.1 & & & & 22.1 \\
\hline Sparidae & C. penna & 12.6 & 83.1 & 72.8 & 72.1 & 0.8 & & & 2.5 & 1.5 & 35.1 \\
\hline Carangidae & C. bartholomaei & 0.4 & & 1.1 & 12.2 & & & & & & 4.6 \\
\hline Haemulidae & H. plumierii & 0.5 & & & & & & & & & 0.5 \\
\hline Palinuridae & P. argus & 1.0 & 1.3 & 1.8 & 17.2 & & & 2.5 & 2.8 & 2.5 & 4.2 \\
\hline Labridae & S. aurofrenatum & & 0.5 & & & & & & & & 0.5 \\
\hline \multirow[t]{2}{*}{ Priacanthidae } & H. cruentatus & & 1.0 & & & & & 0.5 & 5.0 & 1.5 & 2.0 \\
\hline & Total (kg) & 38.7 & 173.8 & 205.5 & 233.43 & 9 & 82.9 & 42.5 & 65.75 & 47.25 & \\
\hline
\end{tabular}

